\title{
On a paper by A. Baker on the approximation of rational powers of $e$
}

by

KURT MAHLER (Canberra)

In an important paper of 1965 (Canadian Journal of Mathematios, 17, pp. 616-626), A. Baker for the first time established lower bounds for products of the form

(I) $\quad\left|x_{1} x_{2} \ldots x_{l k}\left(x_{1} E_{1}+x_{2} E_{2}+\ldots+x_{k} E_{k}\right)\right|$

$$
\text { and } y_{k}\left|y_{k} E_{1}-y_{1}\right| \cdots\left|y_{k} E_{k-1}-y_{k-1}\right| \text {. }
$$

Here $E_{1}, E_{2}, \ldots, E_{k}$ are distinct rational powers of $e$, with $E_{k}=1$ in the second expression; the $x$ 's are distinct integers not zero, while the $y$ 's are integers where $y_{k}>0$, and $k \geqslant 2$. These lower bounds involve positive constants depending only on $k$ and the $E$ 's and are not given explicitly. The method depends on an ingenious generalization of that by C. L. Siegel in his classical paper in the Abhandlungen der Preussischen Akademie der Wissenschaften of 1929 , No. 1.

I try in the present paper to carry Baker's investigations a little further by establishing lower bounds for the expressions (I) which are completely explicit and do not involve any unknown constants; the results are contained in the Theorems $I$ and 2 and their corollaries. It is highly probable that better estimates can be proved if explieit formulae for Baker's approximation polynomials are used. Such formulae have been obtained recently by A. van der Poorten at the University of New South Wales.

1. This paper makes use of the following well known theorem.

LEEMMA 1. Let

$$
\left(g_{i j}\right) \quad(i=1,2, \ldots, M ; j=1,2, \ldots, N),
$$

where $M<N$, be a matrix of integers, and tet

$$
G_{i}=\sum_{j=1}^{N}\left|g_{i j}\right| \quad(i=1,2, \ldots, M)
$$


Then there exist integers $x_{1}, \ldots, x_{N}$ not all zero such that

2. Let $a_{1}, \ldots, a_{k}$, where $k \geqslant 2$, be finitely many distinct integers,

$$
\begin{aligned}
& \sum_{j=1}^{N} g_{i j} x_{j}=0 \quad \text { for } \quad i=1,2, \ldots, M \\
& \max \left(\left|x_{1}\right|, \ldots,\left|x_{N}\right|\right) \leqslant\left(G_{1} \ldots G_{M}\right)^{1 /(N-M)}
\end{aligned}
$$

Proof. Put

$$
G=\left[\left(G_{1} \ldots G_{M}\right)^{1 /(N-M)}\right],
$$

where $[s]$ as usual denotes the integral part of $s$. There are then $(G+1)^{N}$ distinct vectors $x=\left(x_{1}, \ldots, x_{N}\right)$ with integral components $x_{1}, \ldots, x_{N}$ satisfying

$$
0 \leqslant x_{j} \leqslant G \quad(j=1,2, \ldots, N) .
$$

With each such vector $x$ associate a second integral vector $y=\left(y_{1}, \ldots, y_{M}\right)$ where

$$
y_{i}=\sum_{j=1}^{N} g_{i j} x_{j} \quad(i=1,2, \ldots, M)
$$

Further define for each suffix $i=1,2, \ldots, M$ two non-negative integers $n_{i}$ and $p_{i}$ by

$$
n_{i}=\sum_{\substack{j=1 \\ \theta_{i j}<0}}^{N}\left|g_{i j}\right|, \quad p_{i}=\sum_{\substack{j=1 \\ g_{i j}>0}}^{N}\left|g_{i j}\right| \quad(i=1,2, \ldots, M)
$$

Then evidently

$$
G_{i}=n_{i}+p_{i} \quad(i=1,2, \ldots, M)
$$

and for all vectors $y$,

$$
-n_{i} G \leqslant y_{i} \leqslant+p_{i} G \quad(i=1,2, \ldots, M) .
$$

This means that each component $y_{i}$ has at most $n_{i} G+p_{i} G+1=G_{i} G+$ +1 possibilities, hence that the vector $y$ has at most $\left(G_{1} G+1\right) \ldots\left(G_{M} G+1\right)$ possibilities. But

$$
\begin{aligned}
(G+1)^{N} & =(G+1)^{M}(G-1-1)^{N-M}>(G+1)^{M} G_{1} \ldots G_{M I} \\
& \geqslant\left(G_{1} G+1\right) \ldots\left(G_{M} G+1\right)
\end{aligned}
$$

Hence there are more distinct vectors $x$ than there are distinct vectors $y$. It follows that a certain pair of distinct $x$-vectors, $x^{\prime}$ and $\boldsymbol{x}^{\prime \prime}$ say, generate the same vector $y$. This implies that their difference $x=\boldsymbol{x}^{\prime}-\boldsymbol{x}^{\prime \prime}$ is not itself the zero vector, but generates the zero vector $y=(0, \ldots, 0)$. Since the components $x_{1}, \ldots, x_{N}$ of $x$ evidently lie between $-G$ and $+G$, the vector $x$ has the asserted properties.

and let $a$ be a positive integer satisfying

$$
\left(a, a_{1}, \ldots, a_{k}\right)=1
$$

let further

$$
\dot{A}=\max \left(\left|a_{1}\right|, \ldots,\left|a_{k}\right|\right) \text { and } B=A+a
$$

so that

Put

$$
A \geqslant 1, \quad B \geqslant 2
$$

$$
E_{1}=e^{a_{1} / a}, \ldots, E_{k}=e^{a_{k} / a} .
$$

Then $E_{1}, \ldots, E_{k}$ are distinct positive numbers, and hence the exponential functions

$$
E_{1}^{z}, \ldots, E_{k}^{z}
$$

are linearly independent over the field of rational functions of $z$.

Next denote by $r_{1}, \ldots, r_{k}, R$ variable positive integers, and put

$$
\begin{gathered}
r=\max \left(r_{1}, \ldots, r_{k}\right), \quad r_{0}=\min \left(r_{1}, \ldots, r_{k}\right), \\
m=r_{1}+\ldots+r_{k}+k-R, \quad n=r_{1}+\ldots+r_{k i}+k=m+R .
\end{gathered}
$$

It will be assumed that

$k \leqslant R \leqslant r_{1}+\ldots+r_{k}+k-1$, hence that $1 \leqslant m \leqslant r_{1}+\ldots+r_{k} \leqslant k r$.

Since the following three expressions will occur frequently, the following abbreviations will be used,

$$
k^{*}=\frac{k(k-1)}{2}, \quad m^{*}=\frac{m(m-1)}{2}, \quad R^{*}=\frac{1}{R} .
$$

3. With each pair of suffices $(\ddot{i}, j)$ satisfying $1 \leqslant i \leqslant k, j \geqslant 0$ associate two coefficients $p_{i j}$ and $p(i, j)$ related by the equation

$$
p(i, j)=\frac{m !}{j !} p_{i j} .
$$

Both coefficients are assumed equal to zero whenever $(i, j)$ does not belong to the set $S$ of all pairs $(i, j)$ satisfying $1 \leqslant i \leqslant x^{\prime}, r-r_{i} \leqslant j \leqslant r$.

With these coefficients form now the $k$ polynomials

$$
P_{i}(z)=r ! \sum_{j=0}^{r} p_{i j} \frac{z^{j}}{j !}=\sum_{j=0}^{r} p(i, j) z^{j} \quad(i=1,2, \ldots, k)
$$


and the entire function

$$
F(z)=\sum_{i=1}^{k} P_{i}(z) Z_{i}^{z}
$$

say with the power series

$$
F(z)=r ! \sum_{h=0}^{\infty} f_{h} \frac{z^{h}}{h !}
$$

where the coefficients $f_{h}$ are defined by

$$
a^{h} f_{h}=\sum_{i=1}^{k} \sum_{j=0}^{h}\left(\begin{array}{l}
h \\
j
\end{array}\right) a_{i}^{h-j} a^{j} p_{i j} \quad(h=0,1,2, \ldots) .
$$

Denote by $G_{h+1}$ the sum of the absolute values of the coefficients of all the $p_{i j}$ in this equation. Thus

and therefore

$$
G_{h+1}=\sum_{i=1}^{k} \sum_{j=0}^{h}\left(\begin{array}{l}
h \\
j
\end{array}\right)\left|a_{i}\right|^{h-j} a^{j}=\sum_{i=1}^{k}\left(\left|a_{i}\right|+-a\right)^{h}
$$

$$
G_{h+1} \leqslant k B^{h} \quad(h=0,1,2, \ldots),
$$

whence, in particular,

$$
G_{1} \ldots G_{m} \leqslant k^{m} B^{m^{*}}
$$

4. Apply now Lemma 1 to the system of $m$ homogeneotus linear equations

$$
f_{h}=0 \quad(h=0,1, \ldots, m-1)
$$

for the $n$ unknowns $p_{i j}$ for which $(i, j)$ lies in $S$. In the notation of the lemma, $M=m$ and $N=n$, while the maxima $G_{i}$ satisfy the inequalities (2) and (3). Since $n-m=R$, the lemma shows that

There exist integers $p_{i j}$ not all zero, but equal to zero whenever $(i, j)$ does not lie in $S$, such that

(4) $\quad f_{h}=0 \quad$ for $\quad 0 \leqslant \hbar \leqslant m-1 ; \quad \max \left|p_{i j}\right| \leqslant\left(k^{n n} B^{n^{*}}\right)^{R^{n}}$.

Next, in the sum defining $P_{i}(z)$,

$$
\frac{r !}{j !}=\left(\begin{array}{l}
r \\
j
\end{array}\right)(r-j) !
$$

where it suffices to allow $j$ to run over the interval $r-r_{i} \leqslant j \leqslant r$ and therefore

$$
(r-j) ! \leqslant r_{i} !
$$

Since

$$
\sum_{j=0}^{r}\left(\begin{array}{l}
r \\
j
\end{array}\right)=2^{r}
$$

it follows then from (4) that also

$$
\sum_{j=0}^{r}|p(i, j)| \leqslant 2^{r} r_{i} !\left(k^{m} B^{m^{*}}\right)^{R^{*}} \quad(i=1,2, \ldots, k) .
$$

From their construction, the $p(i, j)$ likewise are integers, and they vanish whenever $(i, j)$ does not lie in $s$.

From (1), (2), (4), and the definition of $G_{h+1}$ it finally follows that

$$
\left|f_{h}\right| \leqslant k(B \mid a)^{h}\left(h^{m} B^{m^{*}}\right)^{R^{*}} \quad \text { for } \quad h \geqslant m .
$$

5. By construction, not all the polynomials $P_{i}(z)$ vanish identically. Denote by $i_{1}, \ldots, i_{K}$, where $1 \leqslant K \leqslant k$, all the distinct suffices $i$ for which $P_{i}(z) \neq 0$. Then, by what was said in $\S 2$ about the exponential functions $E_{1}^{s}, \ldots, E_{k}^{z}$, the $\mathbb{K}$ funotions

$$
g_{1}(z)=P_{i_{1}}(z) E_{i_{1}}^{z}, \quad \ldots, \quad g_{K}(z)=P_{i_{K}}(z) E_{i_{K}}^{z}
$$

are linearly independent over the complex number field so that the Wronski determinant

$$
W(z)=\left|\begin{array}{llll}
g_{1}(z) & g_{2}(z) & \ldots & g_{K}(z) \\
g_{1}^{\prime}(z) & g_{2}^{\prime}(z) & \ldots & g_{K}^{\prime}(z) \\
g_{1}^{\prime \prime}(z) & g_{2}^{\prime \prime}(z) & \ldots & g_{K}^{\prime \prime}(z) \\
\ldots \ldots & \ldots & \ldots & \ldots \\
g_{1}^{(K-1)}(z) & g_{2}^{(K-1)}(z) & \ldots & g_{K}^{(K-1)}(z)
\end{array}\right|
$$

does not vanish identically.

Let now $D$ be the differential operator

$$
D=\frac{d}{d z} .
$$

By the definition of $E_{i}$ and by a well known symbolic relation,

$$
g_{l}^{(j)}(z)=\left(\frac{d}{d z}\right)^{j}\left(P_{i_{l}}(z) E_{i_{l}}^{z}\right)=E_{i_{l}}^{z}\left(D+\left(a_{i_{l}}(a)\right)^{j} P_{i_{l}}(z) .\right.
$$

Put therefore

(7) $\quad P_{i j}(z)=\left(D+\left(a_{i} / a\right)\right)^{j} P_{i}(z) \quad(i=1,2, \ldots, k ; j=0,1,2, \ldots)$.

so that

$$
g_{l}^{(j)}(z)=P_{i_{l}, j}(z) E_{i_{l}}^{e} \quad(l=1,2, \ldots, K ; j=0,1,2, \ldots) .
$$

5 - Acta Arithmetica XXVII. 
It follows that

$$
W(z)=\left(E_{i_{1}} E_{i_{2}} \ldots E_{i_{\mathcal{K}}}\right)^{z} \cdot w(z)
$$

where $w(z)$ denotes the new determinant

$$
w(z)=\left|\begin{array}{llll}
P_{i_{1}, 0}(z) & P_{i_{2}, 0}(z) & \ldots & P_{i_{K}, 0}(z) \\
P_{i_{1}, 1}(z) & P_{i_{2}, 1}(z) & \ldots & P_{i_{K}, 1}(z) \\
\ldots \ldots & \ldots & \ldots & \ldots \\
P_{i_{1}, K-1}(z) & P_{i_{2}, K-1}(z) & \ldots & P_{i_{K}, K-1}(z)
\end{array}\right|
$$

which naturally also is not identically zero.

In this determinant $w(z)$ multiply, for $l=1,2, \ldots, \pi$, the $l$ th column by the factor $\mathbb{E}_{i}^{z}$, and afterwards add the 2 nd, 3 rd $, \ldots, K$ th new columns to the first new column. This leads to the formula

because

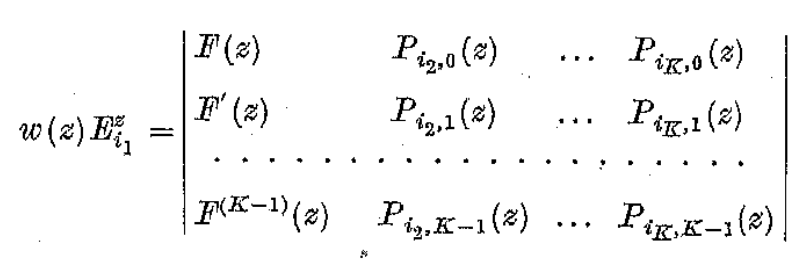

$$
H^{(j)}(z)=\sum_{i=1}^{K} P_{i_{i}, j}(z) E_{i_{l}}^{z} \quad(j=0,1,2, \ldots)
$$

On multiplying in this determinant the successive rows by the factors 1 , $z, z^{2}, \ldots, z^{K-1}$, respectively, we finally arrive at the equation

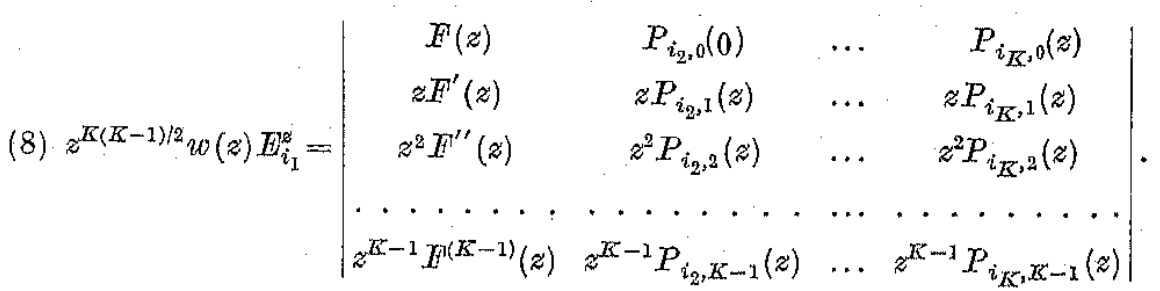

6. By (7), all the $P_{i j}(z)$ are polynomials in $z$ at most of degree $r$, and hence $w(z)$ is a polynomial in $z$ at most of degree K.r. On the other hand, in the determinant (8), all elements of the first column have at $z=0$ a zero at least of order $m$, while, for $l=2,3, \ldots, K$, all elements of the 7 th column have at $z=0$ a zero at least of order $r-r_{i_{l}}$, respectively. Hence $w(z)$ itself has at $z=0$ a zero of order not less than.

$$
\omega=m+\sum_{l=2}^{K}\left(r-r_{i_{l}}\right)-\frac{K(K-1)}{2} .
$$

Since $w(z) \neq 0$ is at most of degree $K r$, it follows that we can write

$$
w(z)=z^{\omega} \Pi(z)
$$

where $\Pi(z) \neq \equiv 0$ is a polynomial in $z$ at most of degree $s=K r-\omega$. Naturally, $s$ cannot be negative.

Let us for the moment, without loss of generality, assume that $r=r_{1}$; this assumption can always be satisfied by a suitable renumbering of the pairs of integers $\left(a_{1}, r_{1}\right), \ldots,\left(a_{k}, r_{k}\right)$. Let us further from now on always assume that
(A)

$$
r_{0} \geqslant R+k^{*}-k+1 \text {. }
$$

The first assumption insures that, in explicit form,

$$
\begin{aligned}
s & =K r-\left(r+\sum_{i=2}^{k} r_{i}+k-R\right)-\sum_{l=2}^{K}\left(r-r_{i_{l}}\right)+\frac{K(K-1)}{2} \\
& =\sum_{l=2}^{K} r_{i_{l}}-\sum_{i=2}^{k} r_{i}-k+R+\frac{K(K-1)}{2} .
\end{aligned}
$$

The hypothesis (A) implies that

$$
K=k \text {. }
$$

For if $K \leqslant l-1$, then there exists a suffix $I$ in the interval $2 \leqslant I \leqslant k$ such that

$$
\sum_{i=2}^{K} r_{i_{L}}-\sum_{i=2}^{l} r_{i} \leqslant-r_{I} \leqslant-R-k^{*}+k-1
$$

and hence it follows from (9) that $s \leqslant-1$ which is absurd.

Since then $K=k$, and since by our notation we may take $i_{1}=1$, we obtain

$$
\sum_{i=2}^{K} r_{i_{l}}=\sum_{i=2}^{l} r_{i}
$$

so that the relation (9) leads to the following result.

LFMma 2. Assume that the condition (A) is satisfied. Then none of the polynomials

$$
P_{1}(z), \ldots, P_{k}(z), \dot{w}(z), \Pi(z)
$$

vanishes identically. Here $w(z)$ is the determinant

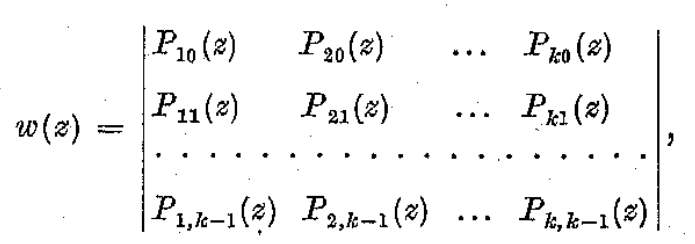




$$
w(z)=z^{\alpha} I I(z)
$$

where $I I(z)$ is a polynomial at most of degree

$$
s=R+k^{*}-k \text {. }
$$

7. The polynomials $P_{i j}(z)$ have been defined by the equations (7). These equations show that they have rational coefficients, hence that the values $P_{i j}(1)$ are rational numbers. In terms of these polynomials, the derivatives

$$
F_{i j}^{(j)}(z)=\sum_{i=1}^{k} P_{i j}(z) E_{i}^{z} \quad(j=0,1,2, \ldots)
$$

are linear forms in the $k$ exponential functions $E_{1}^{\varepsilon}, \ldots, E_{k}^{\varepsilon}$.

By Lemma 2, the determinant $w(z)$ of the first $k$ of these linear forms is not identically zero and has at $z=1$ a zero at most of order $s=R+k^{*}-7$. Let it in fact have a zero of the exact order $\sigma$ so that (10) $w(1)=w^{\prime}(1)=\ldots=w^{(\sigma-1)}(1)=0, w^{(\sigma)}(1) \neq 0$, where $0 \leqslant \sigma \leqslant s$.

On solving the first $k$ linear forms

$$
F^{(j)}(z)=\sum_{i=1}^{h} P_{i j}(z) E_{i}^{z} \quad(j=0,1, \ldots, k-1)
$$

for $E_{i}^{z}$, we obtain equations of the form

$$
w(z) E_{i}^{z}=\sum_{j=0}^{k-1} q_{i j}(z) F^{(j)}(z) \quad(i=1,2, \ldots, k)
$$

where the $q_{i j}(z)$ are cofactors of the determinant $w(z)$ and hence are again polynomials in $z$ with iational coefficients.

Differentiate these $k$ equations $\sigma$ times. Then

$$
\sum_{h=0}^{\sigma}\left(\begin{array}{l}
\sigma \\
h
\end{array}\right) w^{(h)}(z)\left(a_{i} / a\right)^{\sigma-h} E_{i}^{z}=\sum_{j=0}^{k+\sigma-1} Q_{i j}(z) F^{(j)}(z) \quad(i=1,2, \ldots, k)
$$

where also the $Q_{1 j}(z)$ are polynomials in $z$ with rational coefficients.

Here finally put $z=1$. Then, by $(10)$,

$$
w^{(\alpha)}(1) \mathbb{H}_{i}=\sum_{j=0}^{k+\alpha-1} Q_{i j}(1) F^{(j)}(1) \quad(i=1,2, \ldots, k) .
$$

The $k+\sigma$ expressions

$$
\mathbb{F}^{(j)}(1)=\sum_{i=1}^{k} P_{i j}(1) E_{i} \quad(j=0,1, \ldots, k+\sigma-1)
$$

on the right-hand sides of these equations are linear forms in $E_{1}, \ldots, B_{k}$ with rational coefficients, and these $k+\sigma$ linear forms can, by $w^{(\sigma)}(1)$ $\neq 0$, be solved for each of the $E_{i}$.

It follows that there exist $k$ distinet suffices $J=J(1), J(2), \ldots$, $J(k)$ in the interval $0 \leqslant J \leqslant k+s-1=R+k^{*}-1$ for which the corresponding linear forms

$$
F^{(J(j))}(1)=\sum_{i=1}^{k} P_{i, J(j)}(1) E_{i} \quad(j=1,2, \ldots, k)
$$

in $E_{1}, \ldots, E_{k}$ are linearly independent. Hence the determinant of these forms

is distinct from zero.

$$
\Omega=\left|\begin{array}{cccc}
P_{1, J(1)}(1) & \ldots & P_{k, J(1)}(1) \\
\ldots & \ldots & \ldots & \ldots \\
P_{1, J(k)}(1) & \ldots & P_{k, J(k)}(1)
\end{array}\right|
$$

8. The new polynomials

$$
a^{j} P_{i j}(z)=\left(a D+a_{i}\right)^{j} P_{i}(z) \quad(i=1,2, \ldots, k ; j=0,1,2, \ldots)
$$

are again at most of degree $r$, but have integral rather than rational coefficients, say

$$
a^{j} P_{i j}(z)=\sum_{h=0}^{r} p[h, i, j] z^{h} \quad(i=1,2, \ldots, k ; j=0,1,2, \ldots) .
$$

From

$$
P_{i}(z)=\sum_{h=0}^{r} p(i, j) z^{h}
$$

it follows that $a^{j} P_{i j}(z)$ has the explicit form

$$
a^{j} P_{i j}(z)=\sum_{h=0}^{r} \sum_{l=0}^{j}\left(\begin{array}{l}
j \\
l
\end{array}\right) a^{l} a_{i}^{j-l} p(i, h) h(h-1) \ldots(h-l+1) z^{h-l}
$$

Here

$$
(i=1,2, \ldots, k ; j=0,1,2, \ldots) .
$$

$$
\sum_{l=0}^{j}\left(\begin{array}{l}
j \\
l
\end{array}\right) a^{l}\left|a_{i}\right|^{j-l} \leqslant B^{j} \quad \text { and } \quad h(h-1) \ldots(h-l+1) \leqslant h^{l} \leqslant r^{j}
$$

so that

$$
\sum_{h=0}^{r}|p[h, i, j]| \leqslant(r B)^{j} \sum_{h=0}^{r}|p(i, h)|
$$


and therefore, by (5),

$$
\begin{aligned}
&\left.\sum_{h=0}^{r} \mid p[h, i, j]\right\} \leqslant(r B)^{j} 2^{r} r_{i} !\left(k^{m} B^{m^{*}}\right)^{R^{*}} \\
&(i=1,2, \ldots, k ; j=0,1,2, \ldots)
\end{aligned}
$$

Put now

$$
g_{i j}=a^{J(j)} P_{i, J(j)}(1) \quad(i, j=1,2, \ldots, k) .
$$

Then all the numbers $g_{i j}$ are integers, and by $\S 7$ their determinant

$$
g=\left|\begin{array}{ccc}
g_{11} & \ldots & g_{k 1} \\
\ldots & \ldots & . \\
g_{1 k} & \ldots & g_{k j k}
\end{array}\right|=a^{k^{*} \Omega}
$$

does not vanish.

Since none of the suffices $J(j)$ exceeds $R+k^{*}-1$, we deduce immediately from the estimate (12) that

$$
\left|g_{i j}\right| \leqslant C_{1} r_{i} ! \quad\left(i, j=1,2, \ldots, k_{i}\right)
$$

where $O_{1}$ denotes the expression.

$$
C_{1}=2^{r}(r B)^{R+k^{*}-1}\left(k^{m} B^{m^{*}}\right)^{R^{*}}
$$

9. In analogy to the integers $g_{i j}$ put

$$
L_{j}=a^{J(j)} F^{(J(j))}(1) \quad\left(j=1,2, \ldots, j_{i}\right),
$$

so that $L_{j}$ is the linear form

$$
L_{j}=g_{1 j} Z_{1}+\ldots+g_{k j} \mathbb{E}_{k} \quad(j=1,2, \ldots, k)
$$

in $E_{1}, \ldots, E_{k}$. An upper estimate for $\left|L_{j}\right|$ is obtained as follows.

The hypothesis

(A)

$$
r_{0} \geqslant R+\pi^{*}-k+1
$$

implies that

$$
\begin{aligned}
m & =r_{1}+\ldots+r_{k}+k-R \geqslant k\left(R+k^{*}-k+1\right)+k-R \\
& =(k-1) R+(k-2) k^{*}+k,
\end{aligned}
$$

hence, by $k \geqslant 2$, that

(15)

$$
m>R+k^{*}-1 \text {. }
$$

From

$$
F(z)=r ! \sum_{h=m}^{\infty} f_{h} \frac{z^{n^{l}}}{h !}
$$

it follows further that

$$
F^{(j)}(z)=r ! \sum_{h=m}^{\infty} f_{h} \frac{z^{h-j}}{(h-j) !} .
$$

Here we proved already the estimate

$$
\left|f_{h}\right| \leqslant k(B / a)^{h}\left(k^{m} B^{m^{*}}\right)^{R^{*}} \quad \text { for } \quad h \geqslant m .
$$

Hence it follows that

$$
\left|a^{j} F^{(j)}(1)\right| \leqslant a^{j} r ! \sum_{h=m}^{\infty} k(B / a)^{h}\left(k^{m} B^{m^{*}}\right)^{R^{*}} \frac{1}{(h-j) !} .
$$

Here substitute $h=m+l$ in the infinite series; the right-hand side assumes then the form

$$
\frac{a^{j} k r !}{(m-j) !}(B / a)^{m}\left(k^{m} B^{m^{*}}\right)^{R^{*}} \sum_{l=0}^{\infty} \frac{(B / a)^{2}}{(m-j+1)(m-j+2) \ldots(m-j+l)}
$$

where for $j \leqslant m$ the infinite series satisfies the inequality

$$
\sum_{i=0}^{\infty} \leqslant e^{B l a}
$$

Finally let $j$ run over the sulfices $J(1), \ldots, J(k)$. These suffices do not exceed $R+k^{*}-1$, hence by (15) are less than $m$. Thus we obtain the estimate

(16) $\quad\left|L_{j}\right| \leqslant \frac{a^{R+k^{*}-1} e^{B l a} k r !}{\left(m-R-k^{*}-1\right) !}(B \mid a)^{m}\left(k^{m} B^{m^{*}}\right)^{R^{*}} \quad(j=1,2, \ldots, k)$.

Assume now again, just as in $\S 6$, that $r_{1}$ is the largest of the integers $r_{1}, \ldots, r_{k}$, thus that $r=r_{1}$. By $(16)$,

$$
\left|L_{j} r_{2} ! \ldots r_{k} !\right| \leqslant \frac{r_{2} ! \ldots r_{k} !}{\left(m-R-k^{*}-1\right) !} k e^{B / a} a^{R+k^{*}-1-m}\left(k^{m} B^{m^{*}}\right)^{R^{*}} .
$$

Here, by (15),

Further

$$
a^{R+k^{*}-1-m} \leqslant 1
$$

$$
0<m-R-k^{*}-1=\left(r_{1}+\ldots+r_{k}\right)-\left(2 R+k^{*}-k-1\right)
$$

$$
\text { and } r_{i}+\ldots+r_{k} \leqslant 7 r \text {, }
$$

hence

$$
\left(m-R-k^{*}-1\right) ! \geqslant\left(r_{1}+\ldots+r_{k}\right) !(k r)^{-\left(2 R+k^{*}-k-1\right)}
$$


Also

$$
\frac{r_{1} ! \ldots r_{k} !}{\left(r_{1}+\ldots+r_{k}\right) !} \leqslant 1
$$

because the reciprocal of this fraction is an integer. It follows then that

$$
\left|L_{j} r_{2} ! \ldots, r_{k} !\right| \leqslant C_{2} \quad\left(j=1,2, \ldots, l_{0}\right)
$$

where $\mathrm{C}_{2}$ denotes the expression

$$
O_{2}=7 e^{B / a}(k r)^{22 R+k^{*}-k-1} B^{m}\left(k^{m} B^{m^{*}}\right)^{R^{*}} .
$$

The results so proved in this and the preceding section may be combined into the following lemma.

Lemara 3 . Let the notation be as in $\$ 2$ and assume in addition that

$$
r=r_{1} \text { and } r_{0} \geqslant R+k^{*}-k+1 \text {. }
$$

Then there exist to linearly independent linear forms

$$
L_{j}=g_{1 j} E_{1}+\ldots+g_{k j} B_{k i} \quad(j=1,2, \ldots, k)
$$

with integral coefficients $g_{i f}$ such that

$$
\begin{gathered}
\left|g_{i j}\right| \leqslant O_{1} r_{i} ! \quad(i, j=1,2, \ldots, k), \\
\left|\mathcal{L}_{j} r_{2} ! \ldots r_{k_{0}} !\right| \leqslant O_{2} \quad(j=1,2, \ldots, k),
\end{gathered}
$$

where $C_{1}$ and $C_{2}$ are defined by $(14)$ and $(18)$, respectively.

10. Lemma 3 will now be applied to the study of a general linear form. Denote by

$$
L=\infty_{1} E_{1}+\ldots+x_{k} E_{k}
$$

a linear form in $E_{1}, \ldots, E_{k}$ with integral coefficients not all zero, and put

$$
x_{j}^{\prime}=1 \text { if } x_{j}=0, \quad \text { and } \quad x_{j}^{\prime}=x_{j} \text { if } x_{j} \neq 0 \quad\left(j=1,2, \ldots, k_{)},\right.
$$

and

$$
x=\max \left(\left|x_{1}\right|, \ldots,\left|x_{f_{k}}\right|\right)=\max \left(\left|x_{1}^{\prime}\right|, \ldots,\left|x_{k_{k}}^{\prime}\right|\right) .
$$

We shall now choose the parameters $r_{1}, \ldots, r_{k}, R$ of Lemma 3 as functions of $x_{1}^{\prime}, \ldots, x_{k}^{\prime}$ by the following construction.

Put

$$
O=O(r)=k^{2} r((\log B)(\log r))^{1 / 2},
$$

and define a function $f(r)$ of the positive integer $r$ by

$$
f(r)=e^{-2 C(r)} r !
$$

A well known form of Sterling's formula states that

$$
r !=\sqrt{2 \pi r} r^{r} e^{-r+e(r)}, \quad \text { where } \quad 0<\varrho(r)<\frac{1}{12 r} .
$$

It follows that

$$
\frac{\log f(r)}{r}=\log r-2 k^{2}((\log B)(\log r))^{1 / 2}-1+\sigma(r),
$$

where $\sigma(r)$ denotes the expression.

$$
\sigma(r)=\frac{\log r}{2 r}+\frac{\log 2 \pi}{2 r}+\frac{\varrho(r)}{r} .
$$

Here, for $r \geqslant 2$, it is easily verified that

hence that

$$
0<\sigma(r)<1
$$

(19)

$\log r-2 k^{2}((\log B)(\log r))^{1 / 2}-1<\frac{\log f(r)}{r}<\log r-2 k^{2}((\log B)(\log r))^{1 / 2}$.

The definition of $f(r)$ and this inequality show immediately that

$$
f(\mathbb{I})=1 ; \quad f(r)<1 \quad \text { if } \quad 2 \leqslant r \leqslant B^{4 k^{4}} .
$$

It is also obvious that

$$
O(r-1)<O(r) \quad \text { if } \quad r \geqslant 2 .
$$

By definition, $x$ is a positive integer. There exists therefore a smallest positive integer $r$ such that

$$
f(r)>x,
$$

and this integer necessarily has the further properties

so that by (21) also

Define similarly the integers $r_{1}, \ldots, r_{k}$ by the inequalities

$$
\text { (24) } \quad\left(r_{j}-1\right) ! \leqslant e^{2 C(r)}\left|x_{j}^{\prime}\right|<r_{j} ! \quad(j=1,2, \ldots, k) \text {. }
$$

Then by (23) and (24) and in agreement with the hypothesis of $\S 2$,

$$
r=\max \left(r_{1}, \ldots, r_{k}\right) \text {. }
$$

Without loss of generality, let from now on

$$
x=\left|x_{1}^{\prime}\right|=\left|x_{1}\right|
$$


be the largest of the integers $\left|x_{1}^{\prime}\right|, \ldots,\left|x_{k}^{\prime}\right|$. The formulae (23) and (24) imply then that also

$$
r=r_{1}
$$

is the largest of the integers $r_{1}, \ldots, r_{k}$, in agreement with the previous assumption.

By $(22), f(r)$ is greater than $x \geqslant 1$. Hence, by $(20)$ necessarily

$$
r \geqslant B^{4 k^{4}}+1 \text {. }
$$

11. Having fixed $r, r_{1}, \ldots, r_{k}$ in this manner, define now $R$ by

$$
R=\left[\operatorname{ler}\left(\frac{\log B}{\log r}\right)^{1 / 2}\right]+1
$$

so that

$$
\operatorname{kr}\left(\frac{\log B}{\log r}\right)^{1 / 2}<R \leqslant \operatorname{kr}\left(\frac{\log B}{\log r}\right)^{1 / 2}+1 .
$$

By $(25)$ and $k \geqslant 2$ this choice implies that

$$
R<\frac{k}{2 k^{2}} r+1<r+k-1
$$

and since $r(\log r)^{-1 / 2}$ is an increasing function of $r$ when (25) holds, it also follows from $B \geqslant 2$ that

$$
R>\frac{B^{4 k^{4}}}{2 k} \geqslant \frac{2^{4 k^{4}}}{2 k}>\frac{4 k^{4}}{2 k}>\max \left(k, k^{*}\right)
$$

Hence the condition

$$
k \leqslant R \leqslant r_{1}+\ldots+r_{k}+k-1
$$

of $\S 2$ is certainly satisfied. It further follows that

$$
R+k^{*}-k+1<2 R
$$

The former hypothesis

$$
r_{0} \geq R+k^{*}-k+1
$$

does then certainly hold if

$$
r_{j} \geqslant 2 R \quad(j=1,2, \ldots, k) .
$$

That this set of inequalities is in fact satisfied will now be proved indirectly. Assume there exists a suffix $j$ for which

$$
r_{j}<2 R \text {. }
$$

Then, by (26) and (27),

$$
r_{j}<2 R \leqslant 2 k r\left(\frac{\log B}{\log r}\right)^{1 / 2}+2<3 k r\left(\frac{\log B}{\log r}\right)^{1 / 2},
$$

whence

$$
\log r_{j} ! \leqslant r_{j} \log r_{j}<3 k r\left(\frac{\log B}{\log r}\right)^{1 / 2} \log \left(3 k r\left(\frac{\log B}{\log r}\right)^{1 / 2}\right) .
$$

Here, again by $(25)$ and by $k \geqslant 2$,

$$
3 k\left(\frac{\log B}{\log r}\right)^{1 / 2}<\frac{3 k}{2 k^{2}}<1 .
$$

Hence

$$
\log r_{j} !<3 \operatorname{kr}\left(\frac{\log B}{\log r}\right)^{1 / 2} \cdot \log r=3 k r((\log B)(\log r))^{1 / 2},
$$

and so, once more by $k \geqslant 2$,

$$
r_{j} !<e^{2 C(r)}
$$

contrary to the definition (24) of $r_{j}$ because $\left|x_{j}^{\prime}\right|$ is at least 1 .

We have thus proved that the definitions (22), (24), and (26) of $r, r_{1}$, $\ldots, r_{k}$, and $R$, together with a notation such that $x=\left|x_{1}^{\prime}\right|$ and hence also $r=r_{1}$, satisfy all the conditions of $\S 2$ and of Lemma 3 . We are then allowed to apply this lemma.

12. This means that, in addition to the given linear form

$$
L=x_{1} E_{1}+\ldots+x_{k} E_{k}
$$

there exist the $k$ linearly independent linear forms

$$
L_{j}=g_{1 j} E_{1}+\ldots+g_{k j} E_{k} \quad\left(j=1,2, \ldots, l_{i}\right)
$$

of the lemma which have integral coefficients such that

$$
\begin{gathered}
\left|g_{i j}\right| \leqslant C_{1} r_{i} ! \quad(i, j=1,2, \ldots, k), \\
\left|\mathcal{L}_{j} r_{2} ! \ldots r_{k} !\right| \leqslant C_{2} \quad(j=1,2, \ldots, k) .
\end{gathered}
$$

The form $L$ is then linearly independent of certain $k-1$ of the forms $L_{j}$. To fix the ideas, assume that the $k$ forms

$$
L, L_{2}, \ldots, L_{k}
$$

are linearly independent. Hence their determinant

$$
\left|\begin{array}{ccc}
x_{1} & \ldots & x_{k} \\
g_{12} & \ldots & g_{k 2} \\
\cdots & \cdots & \ddots \\
g_{1 k} & \ldots & g_{k k k}
\end{array}\right|,=\Delta \text { say, }
$$


does not vanish. This determinant is an integer, and so

$$
|\Delta| \geqslant 1 \text {. }
$$

On solving the $k$ forms (29), say for $E_{1}$, we find that

It follows that

$$
\left|\begin{array}{cccc}
L_{1} & x_{2} & \ldots & x_{k} \\
L_{2} & g_{22} & \ldots & g_{k 2} \\
\ldots & \cdots & \cdots & \cdot \\
L_{l k} & g_{2 k} & \ldots & g_{l k k}
\end{array}\right|=\Delta E_{1}
$$

$$
\Delta e^{a_{1} / a}=L M+L_{2} M_{2}+\ldots+L_{k} M_{k}
$$

where $M, M_{2}, \ldots, M_{k_{k}}$ denote the cofactors of the elements $L, L_{2}, \ldots, L_{k}$ in the first column of the determinant for $\Delta E_{1}$, respectively.

By (28),

$$
\mid\left[M \mid \leqslant(k-1) ! O_{1}^{T c-1} r_{2} ! \ldots r_{k} !\right.
$$

and since $\left|x_{j}\right| \leqslant\left|x_{j}^{\prime}\right|$,

$$
\left|M_{j}\right| \leqslant(k-2) ! O_{1}^{k-2} O_{2} r_{2} ! \ldots r_{k} ! \sum_{l=2}^{l_{b}} \frac{\left|x_{l}^{\prime}\right|}{r_{l} !} \quad\left(j=2,3, \ldots, k_{k}\right) .
$$

The identity (30) implies therefore the inequality

$$
1 \leqslant U+V
$$

where

(32) $U=(k-1) ! e^{-a_{1} / a} C_{1}^{k-1} r_{2} ! \ldots r_{l l} !|L|, \quad V=(k-1) ! e^{-a_{1} / a} a_{1}^{k-2} O_{2} \sum_{l=2}^{k} \frac{\left|x_{l}^{\prime}\right|}{r_{l} !}$.

We shall next establish upper estimates for $U$ and $\nabla$.

13. Since $r_{j} \leqslant r$ for all $j$, by (24),

$$
r_{2} ! \ldots r_{k} ! \leqslant r^{k-1} e^{2(l-1) C(r)}\left|x_{2}^{\prime} \ldots x_{k_{l}}^{\prime}\right|, \quad \sum_{l=2}^{k} \frac{\left|x_{l}^{\prime}\right|}{r_{l} !} \leqslant(k-1) e^{-2 O(r)},
$$

and therefore

$U \leqslant\left(k_{i}-1\right) ! e^{-a_{1} / a} O_{1}^{k-1} r^{k-1} e^{2(k-1) O(r)}\left|x_{2}^{\prime} \ldots x_{k}^{\prime} X\right|, \quad V \leqslant k ! e^{-a_{1} / a} O_{1}^{k-2} C_{2} e^{-2 O(r}$.

Here, by the definitions (14) and (18),

$$
O_{1}=2^{r}(r B)^{R+l^{*}-1}\left(k^{m} B^{m^{*}}\right)^{R^{*}}, \quad O_{2}=k e^{B / a}(7 c r)^{2 R+k^{*}-k-1} B^{m}\left(k^{m} B^{m^{*}}\right)^{R^{*}} .
$$

It follows that

$$
U \leqslant O_{3}\left|x_{2}^{\prime} \ldots x_{k}^{\prime} L\right|, \quad V \leqslant C_{4}
$$

where $C_{3}$ and $C_{4}$ are defined by

$$
O_{3}=(k-1) ! e^{-a_{1} / a}\left(2^{r}(r B)^{R+k^{*}-1}\left(k^{m} B^{m^{*}}\right)^{R^{*}}\right)^{k-1} r^{k-1} e^{2(k-1) C(r)}
$$

and .

$O_{4}=k ! e^{-a_{1} / a}\left(2^{r}(r B)^{R+k^{*}-1}\left(k^{m} B^{m^{*}}\right)^{R^{*}}\right)^{k-2} k e^{B / a}(k r)^{2 R+k^{*}-k-1} B^{m}\left(k^{m} B^{m^{*}}\right)^{R^{*}} e^{-2 C(r)}$.

Here it is convenient to split off the factors of maximal size from $C_{3}$ and $O_{4}$ and to write these expressions as

(34) $\quad O_{3}=O_{5} e^{2(k-1) C(r)} r^{(l k-1)(R-1)} B^{(k-1) m^{*} R^{*}}, \quad O_{4}=O_{6} e^{-2 C(r)} r^{k(R-1)} B^{(k-1) m^{*} R^{*}}$, where the new factors $O_{5}$ and $O_{6}$ are given by

and

$$
C_{5}=(k-1) ! e^{-a_{1} / a} 2^{(k-1) r} k^{(k-1) m R^{*}} r^{(k-1)\left(k^{*}+1\right)} B^{(k-1)\left(R+k^{*}-1\right)}
$$

$O_{6}=k \cdot k ! e^{\left(B-a_{1}\right) / a_{2}} 2^{(k-2) r} k^{2 R+(k-1) m R^{*}+k^{*}-k-1} r^{(k-1) k^{*}-k+1} B^{(k-2)\left(R+k^{*}-1\right)+m}$.

The next step consists in obtaining simple upper estimates for $2 O_{5}$ and $2 O_{6}$ and hence also for $2 O_{3}$ and $2 C_{4}$.

14. Firstly, by the definition (26) of $R$,

while

$$
R-1 \leqslant 7 r\left(\frac{\log B}{\log r}\right)^{1 / 2} \leqslant R
$$

$$
m \leqslant k r \text { and therefore } m^{*} \leqslant \frac{k^{2} r^{2}}{2} \text {. }
$$

The second factors of $C_{3}$ and $O_{4}$ in (34) have therefore the upper bounds

and

$$
\begin{gathered}
e^{2(k-1) C(r)} r^{(k-1)(R-1)} B^{(k-1) m^{*} R^{*}} \leqslant e^{\left(2 k_{0}-\frac{1}{2}-\frac{3}{2 k}\right) C(r)} \\
e^{-2 C(r)} r^{k(R-1)} B^{(k-1) m^{*} R^{*}} \leqslant e^{-\left(\frac{1}{2}+\frac{1}{2 k}\right) C(r)} .
\end{gathered}
$$

To deal with the first factors $C_{5}$ and $C_{6}$, we first note that

$$
\begin{aligned}
2(k-1) ! \leqslant k^{k}, \quad k \cdot 7 ! \leqslant k^{k}, & (k-1)\left(k^{*}+1\right) \leqslant k^{3} / 2, \\
(k-1) k^{*}-k+1 \leqslant k^{3} / 2, & R+k^{*}-1 \leqslant 2 R
\end{aligned}
$$

and 
Therefore

$$
2 O_{5} \leqslant k^{k} e^{B} 2^{k r} k^{l l^{2} r R^{*}} r^{k k^{3} / 2} B^{2 k R R},=e^{C_{7}} \text { say, }
$$

and.

$$
2 O_{6} \leqslant k^{k} e^{2 B} 2^{k r} k^{2 R+k^{2} r R^{*}+k^{2} / 2} r^{k^{3 / 2} / 2} B^{2 k R+k r},=e^{C_{8}} \text { say. }
$$

Here

$k \geqslant 2, \quad \log k \leqslant e^{-1} k<k, \quad B \geqslant 2, \quad \log B \leqslant e^{-1} B<B, .2 \cdot \log B>1$.

Also, by (25),

$$
r>B^{4 k^{4}} \geqslant 2^{64}>e^{32}, \quad \log r>4 k^{4} \log B>2 k^{4} \geqslant 32 .
$$

Therefore

$$
((\log B)(\log r))^{1 / 2} \geqslant 2 k^{2} \log B>k^{2}
$$

whence

$$
O(r)=k^{2} r((\log B)(\log r))^{1 / 2}>k^{4} r>k^{4} B^{4 k^{4}} \geqslant 2^{68}
$$

We also note that, for the values of $r$ considered, the function

$$
\frac{\log r}{r}
$$

is strictly decreasing and hence satisfies the inequality

$$
\frac{\log r}{r}<\frac{4 k^{4} \log B}{B^{4 k^{4}}}<\frac{4 k^{4}}{B^{4 k^{4}-1}} \leqslant \frac{2^{6}}{2^{63}}=2^{-57} .
$$

Thus the following upper estimates for the successive terms of $C_{7}$ and $C_{\mathrm{s}}$ are obtained.

$$
\begin{gathered}
O(r)^{-1} \cdot k \cdot \log k<\frac{k^{2}}{k^{4} r}=\frac{1}{l^{2} r}<\frac{1}{2^{2} \cdot 2^{64}}=2^{-6 n} \\
O(r)^{-1} \cdot 2 B<\frac{2 B}{k^{4} B^{4 k^{4}}} \leqslant \frac{1}{k^{3} B^{4 k^{4}-1}} \leqslant \frac{1}{2^{3} \cdot 2^{63}}=2^{-66} ; \\
O(r)^{-1} \cdot 7 r \cdot \log 2<\frac{\log 2}{k((\log B)(\log r))^{1 / 2}} \leqslant \frac{\log 2}{k \cdot 2 k^{2} \log B} \leqslant \frac{1}{2 k^{3}} \leqslant \frac{1}{16} \\
O(r)^{-1} \cdot 2 R \cdot \log k<\frac{3 k r\left(\frac{\log B}{\log r}\right)^{1 / 2} \cdot e^{-1} k}{k^{2} r((\log B)(\log r))^{1 / 2}}=\frac{3}{e k \cdot \log r}<\frac{3}{64 e}<\frac{1}{40}
\end{gathered}
$$

$$
\begin{aligned}
C(r)^{-1} \cdot k^{2} r R^{*} \log k & <\frac{k^{2} r \cdot \log k}{k r\left(\frac{\log B}{\log r}\right)^{1 / 2} \cdot k^{2} r((\log B)(\log r))^{1 / 2}} \\
& =\frac{\log k}{k r \cdot \log B} \leqslant \frac{1}{e r \cdot \log 2}<\frac{1}{r}<2^{-64} ;
\end{aligned}
$$$$
C(r)^{-1} \cdot \frac{k^{2}}{2} \log k<\frac{k^{2} \cdot k}{2 k^{4} r}=\frac{1}{2 k r}<2^{-66} ;
$$$$
O(r)^{-1} \cdot \frac{k^{3}}{2} \log r<\frac{k^{3} \log r}{2 k^{4} r}=\frac{\log r}{2 k r} \leqslant \frac{\log r}{4 r}<2^{-59} ;
$$$$
C(r)^{-1} \cdot 2 k r \cdot \log B<\frac{k \cdot 3 k r\left(\frac{\log B}{\log r}\right)^{1 / 2} \cdot \log B}{k^{2} r((\log B)(\log r))^{1 / 2}}-=\frac{3 \cdot \log B}{\log r}
$$$$
<\frac{3 \cdot \log B}{4 k^{4} \cdot \log B}=\frac{3}{4 k^{4}}<\frac{1}{16} ;
$$$$
C(r)^{-1} \cdot k r \cdot \log B=\frac{k r \cdot \log B}{k^{2}((\log B)(\log r))^{1 / 2}}=\frac{1}{k}\left(\frac{\log B}{\log r}\right)^{1 / 2}<\frac{1}{k \cdot 2 k^{2}} \leqslant \frac{1}{16} .
$$

On adding these results it follows at once that

hence that

$$
C_{7}<\frac{1}{4} C(r) \text { and } O_{8}<\frac{1}{4} C(r)
$$

$$
2 O_{5}<e^{\sharp C(r)} \text { and } 2 O_{6}<e^{\sharp C(r)} .
$$

Therefore, by. (34), (35), and (36),

and

$$
\begin{gathered}
2 O_{3}<e^{\left(2 k-\frac{1}{4}-\frac{3}{2 k}\right) C(r)}<e^{\left(2 k-\frac{1}{4}\right) C(r)}<e^{2 k C(r)} \\
2 C_{4}<e^{-\left(\frac{1}{4}+\frac{1}{2 k}\right) C(r)}<e^{-\frac{1}{4} C(r)}<1
\end{gathered}
$$

By (33), these estimates imply that

$$
2 U<e^{2 L C(r)}\left|x_{2}^{\prime} \ldots x_{k}^{\prime} L\right| \text { and } 2 V<1 .
$$

(In fact, they imply the slightly stronger inequalities

(39) $\quad 2 U<e^{(2 k-\xi) C(r)}\left|x_{2}^{\prime} \ldots x_{k}^{\prime} L\right|$ and $2 V<e^{-\sharp C(r)}$.)

Since $2 V<1$, it next follows from (31) that

$$
20 \div>1 \text {. }
$$


This we combine with the upper bound for $2 U$ just obtained, and we multiply both sides of the resulting inequality by the factor $x=\left|x_{1}\right|$. We may then again drop the hypothesis that $\left|x_{1}\right|=\max \left(\left|x_{1}\right|, \ldots,\left|x_{k_{k}}\right|\right)$ and so obtain the following result.

THeosear 1. Let $a_{1}, \ldots, a_{k}$, where $k \geqslant 2$, be distinct integers, and let $a>0$ be an integer satisfying $\left(a, a_{1}, \ldots, a_{k}\right)=1$; let further $x_{1}, \ldots, x_{k}$ be integers not all zero. Put

$$
B=a+\max \left(\left|a_{1}\right|, \ldots,\left|a_{k}\right|\right), \quad \mathbb{E}_{1}=e^{a_{1} / a}, \ldots, \mathbb{B}_{k}=e^{\dot{a}_{k} / a}
$$

and

$$
\begin{gathered}
x_{j}^{\prime}=\left\{\begin{array}{ccc}
1 & \text { if } & x_{j}=0 \\
x_{j} & \text { if. } & x_{j} \neq 0
\end{array} \quad(j=1,2, \ldots, k) ;\right. \\
x=\max \left(\left|x_{1}\right|, \ldots,\left|x_{k \mid}\right|\right) .
\end{gathered}
$$

Also, for positive integral $r$, put

$$
O(r)=k^{2} r((\log B)(\log r))^{1 / 2}, \quad f(r)=e^{-2 \alpha(r)} r !
$$

If $r$ denotes the smallest positive integer for which

then

$$
f(r-1) \leqslant x<f(r)
$$

and

$$
r \geqslant B^{4 k^{4}}+1
$$

$$
\left|x_{1}^{\prime} x_{2}^{\prime} \ldots x_{k}^{\prime}\left(x_{1} E_{1}+x_{2} E_{2}+\ldots+x_{k} E_{k}\right)\right|>x e^{-2 k 0(r)} .
$$

By (39), this inequality may in fact be replaced by

(40) $\quad\left|x_{1}^{\prime} x_{2}^{\prime} \ldots x_{k c}^{\prime}\left(x_{1} E_{1}+x_{2} E_{2}+\ldots+x_{k} E_{k}\right)\right|>x e^{-(2 k--1) C(r)}$,

\section{which is slightly stronger.}

15. As a corollary to this theorem we show how it simplifies when $x$ is very large, thus under the hypothesis that, say

$$
x \geqslant B^{16 i^{4} \cdot B^{16 i^{4}}} \text {. }
$$

It had been found in $\S 10$, formula (19), that

$$
\log r-2 k^{2}((\log B)(\log r))^{1 / 2}-1<\frac{\log f(r)}{r}<\log r-2 k^{2}((\log B)(\log r))^{1 / 2}
$$

Here the right-hand side implies that

$$
f(r) \dot{<} r^{r}
$$

Since $f(r)>x$, it follows therefore from (41) that now

$$
r \geqslant B^{16 k^{4}}+1
$$

hence, by $k \geqslant 2$ and $B \geqslant 2$, that

$$
r>2^{256} \text {. }
$$

By the first lower bound for $r$,

whence, by (19),

$$
(\log B)^{1 / 2} \leqslant \frac{(\log (r-1))^{1 / 2}}{4 k^{2}},
$$

$$
\frac{f(r-1)}{r-1}>\frac{1}{2} \log (r-1)-1
$$

This implies that

$$
\frac{f(r-1)}{r-1}>\frac{r-1}{r}\left(\frac{1}{2} \log (r-1)-1\right)>\frac{1}{3} \log r
$$

as follows from the very large lower bound (42) for $r$. Thus also

$$
f(r-1)>r^{r / 3} \text {. }
$$

The integer $r$ is then connected with $x$ by the inequalities

so that

$$
r^{r / 3}<x<r^{r}
$$

$$
\frac{r}{3} \log r<\log x<r \cdot \log r,
$$

$\log r-\log 3+\log \log r<\log \log x<\log r+\log \log r \leqslant 2 \cdot \log r$.

On the left-hand side, by (42), trivially

so that

$$
\log \log r>\log 3
$$

$$
\log r<\log \log x<2 \cdot \log r .
$$

On combining the last inequalities, it follows then that

$$
\frac{\log x}{\log \log x}<r<\frac{6 \cdot \log x}{\log \log x}
$$

These inequalities combine to the result that

$$
O(r)<6 k^{2}(\log x)\left\{(\log B)(\log \log x)^{-1}\right)^{1 / 2} .
$$

Theorem 1 implies therefore the following corollary. 
Let $a_{1}, \ldots, a_{k}, a, B, E_{1}, \ldots, \mathbb{t}_{k}, x_{1}, \ldots, x_{k}, x_{1}^{\prime}, \ldots, x_{k}^{\prime}$, and $x$ be as in Theorem 1 , but assume that now

$$
x \geqslant B^{16 k^{4} \cdot B^{16 k^{4}}} \text {. }
$$

Then

$$
\left|x_{1}^{\prime} \ldots x_{k}^{\prime}\left(x_{1} E_{1}+\ldots+x_{k} E_{k}\right)\right|>x \cdot e^{\left.-122 k^{3}(\log x)(\log B)(\log \log x)^{-1}\right)^{1 / 2}} .
$$

This, is Baker's first result, but with explicit constants.

16. Let $a_{1}, \ldots, a_{k}, a, E_{1}, \ldots, E_{k}$ be as in Theorem 1 , but assume that $a_{k}$ and $E_{k}$ have now been specialized by taking

$$
a_{k}=0 \text {, hence } \mathbb{E}_{k_{s}}=1 .
$$

Denote by $y_{1}, \ldots, y_{k}$ positive integers such that

and that the product

$$
y_{k} \geqslant k
$$

$$
\omega=y_{k}\left|y_{k} E_{1}-y_{1}\right| \ldots\left|y_{k} E_{k-1}-y_{k-1}\right|
$$

satisfies the inequality

$$
0<\omega<1 .
$$

Theorem 1 will enable us to establish a lower bound for $\omega$ in terms of $y_{k}$. For this purpose put

$$
\varphi_{j}=\omega^{1 /(k-1)}\left|y_{k} E_{j}-y_{j}\right| \quad(j=1,2, \ldots, k-1)
$$

and assume, without loss of generality, that the notation is such that

Since evidently

$$
\varphi_{1} \geqslant \varphi_{2} \geqslant \ldots \geqslant \varphi_{k-1}>0 \text {. }
$$

$$
\varphi_{1} \varphi_{2} \ldots \varphi_{k-1}=y_{k} \geqslant k \geqslant 2
$$

not all the $p_{j}$ ean be $\leqslant 1$.

$$
\varphi_{k-1}>1 \text { and hence } \varphi_{j}>1 \text { for } j=1,2, \ldots, k-1,
$$

put $x=k-1$; otherwise denote by $x$ the smallest suffix in the interval

for which

$$
1 \leqslant x \leqslant k-2
$$

$$
\varphi_{x+1} \varphi_{x+2} \cdots \varphi_{k-1} \leqslant 1 .
$$

By (45), such a suffix certainly exists.
17. Having fixed $x$ in this way, consider now the system of $x+1 \leqslant k$ linear inequalities,

$$
\begin{gathered}
\left|x_{j}\right| \leqslant \varphi_{j} \quad(j=1,2, \ldots, x-1) \\
\left|x_{x}\right| \leqslant \varphi_{x} \varphi_{x+1} \ldots \varphi_{k-1} \leqslant \varphi_{x} \\
\left|x_{1} y_{1}+\ldots+x_{x} y_{x}+x_{k} y_{k}\right|<1
\end{gathered}
$$

for $x_{1}, x_{2}, \ldots, x_{x}, x_{k}$. The $x+1$ linear forms

$$
x_{1}, x_{2}, \ldots, x_{*}, x_{1} y_{1}+\ldots+x_{*} y_{x}+x_{k} y_{k}
$$

in $x_{1}, x_{2}, \ldots, x_{x}, x_{k}$ on the left-hand sides in (46) have the determinant $y_{k}$; and the product of the right-hand sides is by (45) equal to the same value since

$$
\varphi_{1} \ldots \varphi_{x-1} \cdot \varphi_{x} \varphi_{x+1} \ldots \varphi_{k-1}=y_{k} .
$$

Hence, by Minkowski's theorem on linear forms, the inequalities (46) can be satisfies by a system of $x+1$ integers $x_{1}, x_{2}, \ldots, x_{x}, x_{k}$ not all zero. But since all the $x$ 's and $y$ 's are integers, the last inequality (46) implies the equation

$$
x_{1} y_{1}+\ldots+x_{k} y_{k}+x_{k} y_{k}=0 .
$$

Hence it follows that already at least one of the integers

$$
x_{1}, x_{2}, \ldots, x_{*}
$$

does not vanish. On the other hand, it is uncertain, and in fact of no importance, whether $x_{l c}$ is or is not equal to zero.

We denote from now on by $i_{1}, i_{2}, \ldots, i_{K}$ all the distinct suffices

for which

$$
1,2, \ldots, x
$$

here naturally

$$
x_{i_{l}} \neq 0 \quad(l=1,2, \ldots, K)
$$

$$
1 \leqslant K \leqslant x
$$

18. The right-hand sides $\varphi_{j}$ and $\varphi_{x} \varphi_{x+1} \ldots \varphi_{k-1}$ of the first $*$ inequalities (46) all are greater than 1 . It follows therefore from these inequalities and from the equation (45) that

$$
\left|x_{i_{1}} x_{i_{2}} \ldots x_{i_{K}}\right| \leqslant \varphi_{1} \varphi_{2} \ldots \varphi_{x-1} \cdot \varphi_{*} \varphi_{x+1} \ldots \varphi_{k-1}=y_{k} .
$$

It is also possible to give an upper bound for $x_{k}$. For identically,

$$
x_{1} y_{1}+\ldots+x_{\varkappa} y_{x}+x_{c} y_{l_{c}}=\left(x_{1} E_{1}+\ldots+x_{\varkappa} E_{\varkappa}+x_{k}\right) y_{k}-\sum_{j=1}^{\infty} x_{j}\left(y_{k} \mathbb{E}_{j}-y_{j}\right)
$$


so that, by (47),

$$
\left(x_{1} E_{1}+\ldots+x_{x} E_{x}+x_{k}\right) y_{k}=\sum_{j=1}^{\varkappa} x_{j}\left(y_{k} E_{j}-y_{j}\right)
$$

Here

$$
\left|x_{j}\right| \leqslant \varphi_{j}, \quad\left|y_{k} E_{j}-y_{j}\right|=\frac{\omega^{1 /(k-1)}}{\varphi_{j}} \quad(j=1,2, \ldots, x) .
$$

It follows then from (49) that

$$
\left.\left|x_{1} D_{1}+\ldots+x_{x} E_{x}+x_{k}\right| \leqslant x \cdot \omega\right)^{I /(k-1)} .
$$

Eere $x \leqslant k-1, \omega<1$, and $y_{k} \geqslant \pi$, so that also

$$
\left|x_{1} E_{1}+\ldots+x_{x} E_{x}+x_{k}\right| \leqslant \frac{(k-1) \omega^{1 /(k-1)}}{y_{k}}<1 .
$$

Thus

where

$$
\left|x_{l c}\right|<1+\left|x_{1}\right| E_{1}+\ldots+\left|x_{x}\right| E_{x}
$$

$$
E_{j}=e^{a_{j} / a} \leqslant e^{A / a} \leqslant e^{B} \quad(j=1,2, \ldots, x) .
$$

Hence, by $x \leqslant k-1$,

$$
\left|x_{k}\right|<k e^{B} \max \left(\left|x_{1}\right|, \ldots,\left|x_{x}\right|\right)=k e^{B} \max \left(\left|x_{i_{1}}\right|, \ldots,\left|x_{i_{\mathbb{R}}}\right|\right) .
$$

Therefore, on noting that $x_{x+1}=x_{x+2}=\ldots=x_{l k-1}=0$ and putting

$$
x=\max \left(\left|x_{1}\right|, \ldots,\left|x_{k}\right|\right)=\max \left(\left|x_{i_{1}}\right|, \ldots,\left|x_{i_{K}}\right|,\left|x_{k}\right|\right),
$$

it has been proved that

$$
x<k e^{B} \max \left(\left|x_{i_{1}}\right|, \ldots,\left|x_{i_{K}}\right|\right) .
$$

All factors of the product $x_{i_{1}} x_{i_{2}} \ldots x_{i_{K}}$ aire integers not zero so that the absolute value of this product cannot be less than $\max \left(\left|x_{i_{1}}\right|, \ldots,\left|x_{i_{K}}\right|\right)$. The inequality (51) implies therefore that

$$
\left|x_{i_{1}} x_{i_{2}} \ldots x_{i_{K}}\right| \geqslant\left(7 e^{B}\right)^{-1} x,
$$

and hence it follows from (48) that

$$
x \leqslant k e^{B} y_{k}
$$

Put now again

$$
x_{j}^{\prime}=\left\{\begin{array}{lll}
1 & \text { if } & x_{j}=0 \\
x_{j} & \text { if } & x_{j} \neq 0
\end{array} \quad(j=1,2, \ldots, k\rangle .\right.
$$

Since $\left|x_{k}\right| \leqslant x$, by $(48)$,

whence, by $(50)$,

$$
\left|x_{1}^{\prime} x_{2}^{\prime} \ldots x_{k}^{\prime}\right| \leqslant x y_{k}
$$

(53) $\quad \mid x_{1}^{\prime} x_{2}^{\prime} \ldots x_{k}^{\prime}\left(x_{1} E_{1}+\ldots+x_{k-1} z_{k-1}+x_{k} \mid \leqslant(k-1) x \omega^{1 /(k-1)}\right.$.

19. Apply now to this inequality ( $\tilde{5} 3)$ the remark to Theorem 1. For this purpose, with a slight change of notation, denote by $r^{\prime}$ and $r^{\prime \prime}$ the smallest positive integers satisfying

$$
f\left(r^{\prime}-1\right) \leqslant x<f\left(r^{\prime}\right) \text { and } f\left(r^{\prime \prime}-1\right) \leqslant y_{k}<f\left(r^{\prime \prime}\right),
$$

respectively. It follows immediately from the estimate (40) of $\S 14$ that

$$
(k-1) \omega^{1 /(k-1)}>e^{-\left(2 k-\frac{1}{4}\right) C\left(r^{\prime}\right)}
$$

so that, by the definition of $\omega$,

(54) $\quad y_{k}\left|y_{k} E_{1}-y_{1}\right| \ldots\left|y_{k} E_{k-1}-y_{k-1}\right|>(k-1)^{-(k-1)} e^{-(k-1)\left(2 k-\frac{1}{4}\right) C\left(r^{2}\right)}$.

This formula has still the disadvantage of involving the integer $r^{\prime}$ depending on $x$ rather than the integer $r^{\prime \prime}$ which depends on $y_{k}$. We show now how to change over to a formula involving $r^{\prime \prime}$.

For the moment, put

$$
\beta=2 k^{2}(\log B)^{1 / 2} .
$$

In $\S 10$ we had for every integer $r \geqslant 2$ obtained the formula

$$
\frac{\log f(r)}{r^{*}}=\log r-\beta(\log r)^{1 / 2}-1+\sigma(r),
$$

where

$$
\sigma(r)=\frac{\log (2 \pi r)}{2 r}+\frac{\varrho(r)}{r} \text { and } 0<\varrho(r)<\frac{1}{12 r} .
$$

Assume now again that

$$
r \geqslant B^{4 / h^{4}}+1
$$

then, by $\$ 14$,

$$
r>2^{64}, \quad \log r>\beta^{2}>32, \quad \frac{\log r}{r}<2^{-57} .
$$

From these estimates it is easily deduced that

$$
0<\sigma(r)<\frac{\log r}{r}<2^{-57}
$$


and that therefore

$$
\frac{\log f(r)}{r}<\log r-\beta(\log r)^{1 / 2}-1+2^{-57} .
$$

Henoe, whenever $f(r)>1$, then necessarily

$$
\log r-\beta(\log r)^{1 / 2}>1-2^{-57}>\frac{3}{4} .
$$

On the other hand, from the definition of $f(r)$,

$$
\frac{f(r+1)}{f(r)}=e^{\log (r+1)-\beta(\log (r+1))^{1 / 2}} \cdot e^{-\beta r\left(\log [(r+1]]^{1 / 2}-\log r\right)^{1 / 2)}}
$$

Here, by (55) applied to $r+1$ instead of $r$, the first exponential factor on the right-hand side is greater than $e^{3 / 4}$. Next, by the mean value theorem of differential calculus,

$$
(\log (r+1))^{1 / 2}-(\log r)^{1 / 2}<\left(2 r(\log r)^{1 / 2}\right)^{-1},
$$

hence, by $\log r>\beta^{2}$,

$$
\beta r\left((\log [r+1])^{1 / 2}-(\log r)^{1 / 2}\right)<\frac{\beta}{2 \beta}=\frac{1}{2}
$$

so that the second exponential factor is greater than $e^{-1 / 2}$. We have thus found the basic inequality

$$
f(r+1)>e^{1 / 4} f(r) \quad \text { if } \quad r \geqslant B^{4 k^{4}}+1
$$

20. This inequality shows that $f(r)$ is strictly increasing and that for every pair of positive integers $n$ and $r \geqslant B^{4 k^{4}}+1$,

$$
f(r+n)>e^{n / 4} f(r) \text {. }
$$

Now, by (52) and by the definitions of $r^{\prime}$ and $r^{\prime \prime}$,

$$
f\left(r^{\prime}-1\right) \leqslant k e^{B} y_{l^{k}} \text { and } y_{l k}>f\left(r^{\prime \prime}\right) .
$$

It follows that

$$
r^{\prime}<r^{\prime \prime}+4 B+4 \cdot \log 7+-1
$$

The right-hand side of the estimate (54) is then greater than

$$
(k-1)^{-(k-1)} e^{-(k-1)\left(2 k-\frac{1}{4}\right) C\left(r^{\prime \prime}+4 B+4 \cdot \log k_{k+1}\right)},=M \text { say, }
$$

for $O(r)$ trivially is an increasing function of $r$.
The quantity $4 B+4 \cdot \log k+1$ is negligibly small compared with $r^{\prime \prime} \geqslant B^{4 k^{4}}+1$. From the definition.

$$
C(r)=k^{2} r((\log B)(\log r))^{1 / 2}
$$

of $C(r)$ we deduce then easily that

$$
\|>e^{-2 i k(h-1) C\left(r^{\prime \prime}\right)} .
$$

Hence, on writing again $r$ for $\dot{r}^{\prime \prime}$, the following result has been established.

TheOREM 2. Let $a_{1}, \ldots, a_{k-1}$, where $k \geqslant 2$, be distinct non-vanishing integers, and let $a>0$ be an integer satisfying $\left(a, a_{1}, \ldots, a_{k-1}\right)=1$; let further $y_{1}, \ldots, y_{k}$ be integers such that $y_{k} \geqslant k$. Put

$$
B=a+\max \left(\left|a_{1}\right|, \ldots,\left|a_{k-1}\right|\right), \quad \mathbb{E}_{1}=e^{a_{1} / a}, \ldots, E_{k-1}=e^{a_{k-1} / a}
$$

and define $C(r)$ and $f(r)$ as in Theorem 1 . If $r$ is the smallest positive integer satisfying

then

$$
f(r-1) \leqslant y_{k}<f(r)
$$

and

$$
r \geqslant B^{4 k^{4}}+1
$$

$$
y_{k}\left|y_{k} E_{1}-y_{1}\right| \ldots\left|y_{k} E_{k-1}-y_{k-1}\right|>e^{-2 k(k-1) C(r)} .
$$

21. Considerations similar to those of $\S 15$ allow to replace this estimate by one which, although less good, is more explicit.

We now assume that

$$
y_{k} \geqslant B^{16 k^{4 \cdot} \cdot B^{16 k^{4}}}
$$

Under the same hypothesis as in Theorem 2 it follows then that

$$
y_{k}\left|y_{k} E_{1}-y_{1}\right| \ldots\left|y_{k} E_{k-1}-y_{k-1}\right|>y_{l_{k}}^{-12 k^{3}(k-1)\left(\log B k \log \log y_{k}\right)^{-1 / j^{1 / 2}}} .
$$

Apart from the explicit constants, this estimate is again due to Baker.

It is highly probable that the constants in Theorem 2 and in this corollary can be improved by a direct application of Lemma 3 instead of the transfer method.

MEATHEMATICS DIPARTMENT

INSTITUTE OF ADVANCED STUDIRS

AUSTRALIAN NATTONAY UNIVERSITY

Canberra, ACT., Australia 